\title{
Commercial Gold Nanoparticles on Untreated Aluminum Foil: Versatile, Sensitive, and Cost-Effective SERS Substrate
}

\author{
Kristina Gudun, Zarina Elemessova, Laura Khamkhash, \\ Ekaterina Ralchenko, and Rostislav Bukasov \\ Chemistry Department, SST Nazarbayev University, 53 Kabanbay Batyr Ave, Block 7, Astana, Kazakhstan \\ Correspondence should be addressed to Rostislav Bukasov; rostislav.bukasov@nu.edu.kz
}

Received 15 February 2017; Revised 17 March 2017; Accepted 2 April 2017; Published 27 April 2017

Academic Editor: Nathan C. Lindquist

Copyright (C) 2017 Kristina Gudun et al. This is an open access article distributed under the Creative Commons Attribution License, which permits unrestricted use, distribution, and reproduction in any medium, provided the original work is properly cited.

\begin{abstract}
We introduce low-cost, tunable, hybrid SERS substrate of commercial gold nanoparticles on untreated aluminum foil (AuNPs@AlF). Two or three AuNP centrifugation/resuspension cycles are proven to be critical in the assay preparation. The limits of detection (LODs) for 4-nitrobenzenethiol (NBT) and crystal violet (CV) on this substrate are about $0.12 \mathrm{nM}$ and $0.19 \mathrm{nM}$, respectively, while maximum analytical SERS enhancement factors (AEFs) are about $10^{7}$. In comparative assays LODs for CV measured on AuNPs@Au film and AuNPs@glass are about $0.35 \mathrm{nM}$ and 2nM, respectively. The LOD for melamine detected on AuNPs@Al foil is 27 ppb with 3 orders of magnitude for linear response range. Overall, AuNPs@AlF demonstrated competitive performance in comparison with AuNPs@ Au film substrate in SERS detection of CV, NBT, and melamine. To check the versatility of the AuNPs@AlF substrate we also detected $\mathrm{KNO}_{3}$ with LODs of $0.7 \mathrm{mM}$ and SERS EF around $2 \times 10^{3}$, which is on the same order with SERS EF reported for this compound in the literature.
\end{abstract}

\section{Introduction}

Since SERS was reported about 40 years ago, this label-free, sensitive, and rapid method with significant, multiplexing potential has been in focus of expanding scientific investigation $[1,2]$. SERS has been established as a versatile analytical technique with applications in sensing of explosives, environmental pollutants, and chemical warfare agents $[3,4]$. Applications of SERS in biosensing include, for instance, detection of sugar in blood, enzymes, and DNA, and early medical diagnostics of major health threats for humans (e.g., cancer, tuberculosis, and viruses) and animals $[5,6]$. As one of the most sensitive analytical techniques for identification of molecular species, SERS has demonstrated the capability for single molecule detection, including detection of a single biomolecule $[7,8]$.

SERS is usually performed on silver, gold, and less frequently copper surfaces, which are more susceptible to corrosion $[9,10]$. Still silver and even gold nanoparticles may change shape after just a few hours of exposure to solvents or solutions of thiols, which affects their plasmonic properties $[11,12]$. Another problem is that the most gold and silver SERS substrates with high sensitivity and acceptable reproducibility are still costly for routine SERS detection. For instance, the price for commercially available SERS nanostructured gold substrate "Klarite" was about 100 USD for a single $2 \times 2 \mathrm{~mm}$ SERS active address.

Aluminum, which is undoubtedly much less costly than silver or gold, has been shown as effective plasmonic material in UV-blue light range for LSPR and surface-enhanced fluorescence [13-15]. Computationally and experimentally $\mathrm{Al}$ is shown as SERS active material in UV spectral range [1618]. There is $\mathrm{Al}_{2} \mathrm{O}_{3}$ film formed on the metal surface, which typically has a thickness of $2.5-3 \mathrm{~nm}$, and LSPR position is red-shifted in the presence of this layer [14]. Aluminum is highly reflective material with more than $85 \%$ reflection efficiency in visible range, but until recently $\mathrm{Al}$ had not been known as effective plasmonic material in this range. That is attributed to an existence of interband transition around $800 \mathrm{~nm}(1.5 \mathrm{eV})$ resulting in a large imaginary part of the dielectric function in visible range [19]. However, recently the first SERS phenomenon has been reported on nanostructured (annealed) $\mathrm{Al}$ film with excitation in visible range (e.g., with $785 \mathrm{~nm}$ laser) by Mogensen group [20]. In 
this study as few as $3 \times 10^{5}-3 \times 10^{7}$ molecules of Raman active molecules (adenine, p-mercapto benzoic acid (pMBA), and 4-aminothiophenol (4-ATP)) in the probed focused laser beam area $\left(\sim 1 \mu \mathrm{m}^{2}\right)$ were detected by SERS, but no estimates of SERS EFs or/and LOD were reported. Very recently, it was reported that the average $\mathrm{EF}$ for single silver nanoparticles (AgNPs) modified with 2-methoxythiophenol (MOTP) and drop-casted on gold film is just about twice as high as average EF of MOTP at AgNPs on Al film $\left(5.0 \times 10^{6}\right.$ versus $\left.2.2 \times 10^{6}\right)$ [21]. Another group reported SERS of Rhodamine 6G, Rose Bengal, and crystal violet on the substrate of concave gold nanocubes on Al-6063 alloy [22].

Hybrid SERS substrates can achieve LODs or EFs that would match or exceed those sensing parameters obtained on gold/silver films or even micro/nanostructured SERS substrates. For instance, synthesized silver nanoparticles over polyvinylidene difluoride (PVDF) membrane detected food contaminant melamine with LOD $50 \mathrm{nM} / 6 \mathrm{ppb}$ [23]. Hybrid substrates consisting of gold nanoparticles on microstructured gold surface (Klarite) demonstrated about 3 orders of magnitude higher EFs for detection of 4-ATP relative to EFs on the Klarite surface [24]. SERS on some hybrid substrates can achieve relatively high EFs $\left(10^{7}\right)$ as exemplified in detection of tyrosine in aqueous solution on silver nanoparticle doped filter paper [25]. High accuracy and precision are reported for detection of three drugs $(0.1 \mathrm{mM}$ solutions) on silver spotted two-pence coins by Goodacre group. To detect those drugs in solutions, small volumes of the solution were spotted on the individually prepared silver targets at coin surface and let to dry before the Raman measurements [26].

In this paper we present inexpensive and rapid method for the fabrication of hybrid SERS substrate from commercial gold nanoparticles drop-casted on untreated and unmodified Al foil. Al foil is universally available, low-cost substrate, which is just rinsed with DI water before preparation. The only equipment needed for preparation is minicentrifuge used for 20-30 minutes. The cost of nanoparticles, needed for preparation of $1 \mathrm{~mm}^{2}$ of this SERS active surface, is less than 2 cents. Untreated $\mathrm{Al}$ foil by itself, used as a substrate without gold nanoparticles, demonstrated relatively small maximum analytical enhancement factors (80-240). However, the composite substrate of AuNP@Al foil has about $\times 10^{5}$ higher $\left(10^{7}\right.$ versus $10^{2}$ ) maximum analytical enhancement factors and 4 or 5 orders of magnitude lower LOD relative to LOD for crystal violet $(\mathrm{CV})$ on bare $\mathrm{Al}$ foil substrate $(\sim 0.35 \mathrm{nM} \mathrm{pM}$ versus $\sim 0.01 \mathrm{mM})$. Finally, we will demonstrate versatility of this substrate in SERS detection of such chemicals as 4nitrobenzenethiol (NBT), $\mathrm{KNO}_{3}$, and melamine.

Crystal violet $(\mathrm{CV})$ is a typical synthetic basic cationic dye, which has been widely used in the aquaculture as a veterinary medicine, additive to poultry food to inhibit propagation of harmful bacteria [27]. 4-Nitrobenzenethiol (NBT) is one of the most commonly applied Raman markers, which is used to prepare extrinsic Raman label preparation (ERLs) in sandwich SERS immunoassays for cancer and tuberculosis $[28,29] . \mathrm{KNO}_{3}$ is a major component of self-made explosives, which was recently detected on nanostructured substrate Klarite with maximum SERS EF calculated [30]. Melamine is a toxic compound that has been found in many samples of chicken eggs, dry milk, or baby formula resulting in hundreds of thousands of hospitalizations and hundreds fatalities, especially among children [31].

\section{Experimental}

2.1. Chemicals and Consumables. Gold nanoparticles of various diameters $(40,60$, and 80$), \mathrm{OD}=1$ in PBS buffers obtained from Sigma-Aldrich. Crystal violet (CV), 4nitrobenzenethiol (NBT), melamine, and potassium nitrate obtained from Sigma-Aldrich as well. Al foil was purchased in a supermarket. DI water was obtained from Milli-Q system.

2.2. Instrumentation. The Raman measurements were performed with $785 \mathrm{~nm}$ diode and $633 \mathrm{~nm} \mathrm{He}-\mathrm{Ne}$ lasers on LABRAM Horiba microscope and $\times 50$ objective. The instrument was calibrated with the Raman signal from the principal Raman vibration of silicon centered at $520 \mathrm{~cm}^{-1}$. For detection of all chemicals, the laser power was set to $\sim 1.5 \mathrm{~mW}$ for $633 \mathrm{~nm}$ excitation and $\sim 3 \mathrm{~mW}$ for $785 \mathrm{~nm}$ excitation at the sample position, and the exposure times for each SERS measurement were set to be 1-3 s. Background corrected spectral intensities obtained from 4 or 8 maps were taken in different locations, but within $0.5-1.5 \mathrm{~mm}$ from the center of each address. They were averaged (at least 64 spectra in each map) for each sample.

The AFM measurements were done with AIST AFM in tapping mode to obtain at least 3-7 AFM maps $(10 \times 10$ or $7 \times 7$ or $4 \times 4 \mu \mathrm{m}$ ) with total of hundreds AuNPs for each representative sample.

2.3. Preparation of Substrates. Al substrate was prepared by attaching $\mathrm{Al}$ foil on the glass with a double-sided scotch. We prepared analyzed solutions of different concentrations. NBT and $\mathrm{CV}$ were dissolved in acetonitrile; melamine and $\mathrm{KNO}_{3}$ are dissolved in DI water. Standard solutions with various concentrations were obtained by diluting the stock solution. We used parafilm with perforated holes to limit spreading of the drop by its hydrophobic surface. After washing the addresses with DI water, we applied $25 \mu \mathrm{L}$ of prepared AuNPs solution on the addresses of $5.5 \mathrm{~mm}$ diameter. The suspensions of AuNPs of two diameters (40 and $60 \mathrm{~nm}$; $\mathrm{OD}=1$ in PBS buffers obtained from Sigma-Aldrich) were centrifuged ( $40 \mathrm{~nm}$ AuNPs at $5000 \mathrm{~g}$ for $12 \mathrm{~min}$ and $80 \mathrm{~nm}$ AuNPs at $2500 \mathrm{~g}$ for $10 \mathrm{~min}$, resp.) and then after removal of supernatant they were resuspended in DI water. Typically those cycles were repeated 3 times and suspensions were briefly sonicated ( $3-5 \mathrm{~min})$ before and after each cycle. After solution of AuNP had dried on the substrate, we drop-casted $25 \mu \mathrm{L}$ of analyzed solutions on each address. As additional control we prepared some samples for detection of CV and NBT drop-casting AuNPs onto the same area on the surface of commercial evaporated gold film (100 nm thickness) or glass microscope slides.

2.4. Calculation of Enhancement Factors (EFs) and Limits of Detection (LODs). In order to quantify the SERS signal 


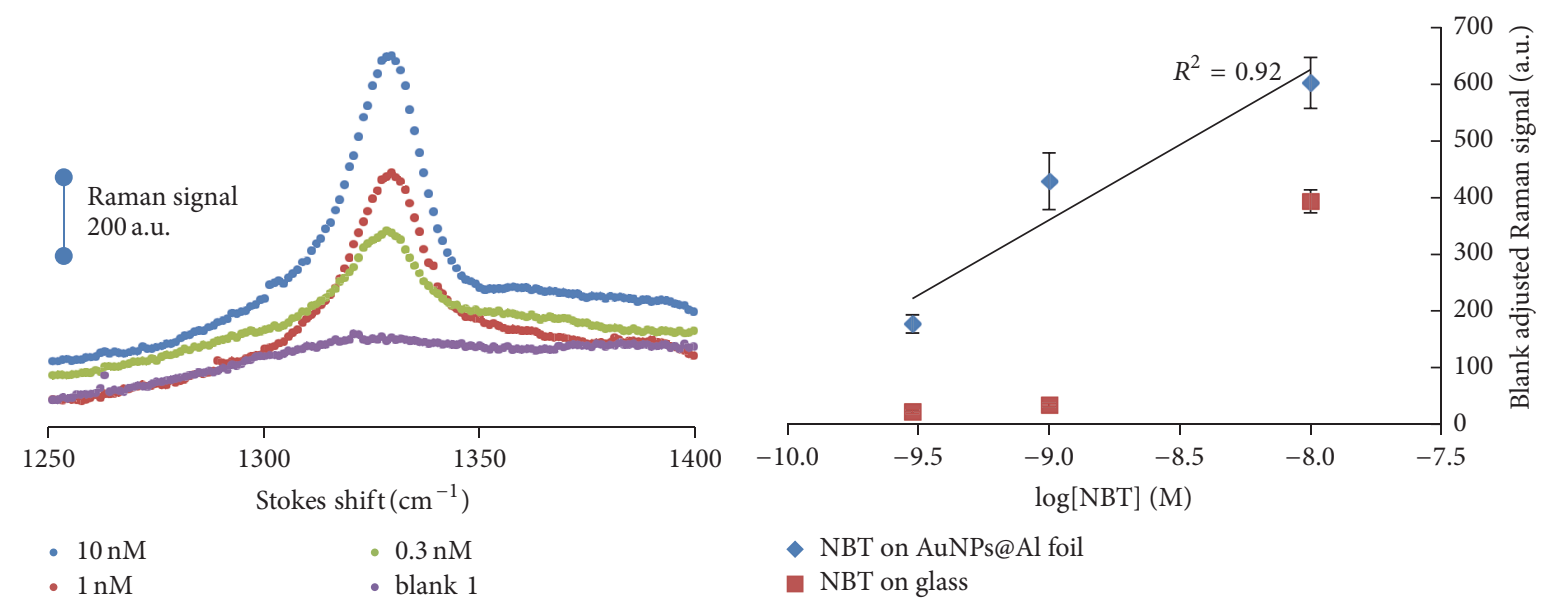

(a)

(b)

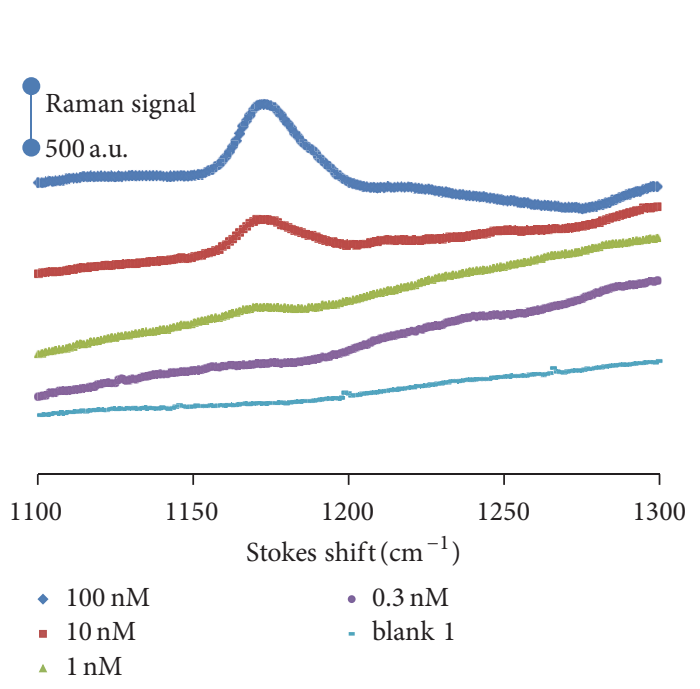

(c)

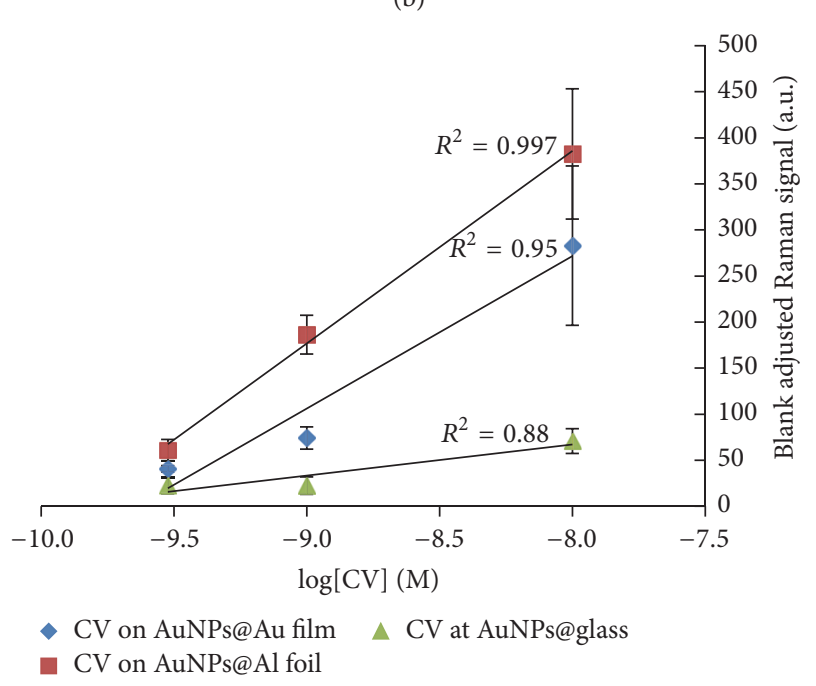

(d)

FIGURE 1: ((a) and (c)) Raman spectra from NBT and CV solutions of different concentrations drop-casted on 60 nm AuNP@Al foil substrate. Spectra are taken with $633 \mathrm{~nm}$ laser excitation. ((b) and (d)) Plots of blank adjusted Raman signal versus logarithm of NBT and CV concentration (M), respectively. Signals are obtained in SERS assay on $60 \mathrm{~nm}$ AuNPs at various substrates.

enhancement capability of the new SERS substrates, the enhancement factor (EF) value is calculated according to the following formula (1):

$$
\mathrm{EF}=\frac{\left(I_{\mathrm{SERS}} / N_{\mathrm{SERS}}\right)}{\left(I_{\mathrm{RAMAN}} / N_{\mathrm{BULK}}\right)}=\frac{I_{\mathrm{SERS}} N v_{\mathrm{BULK}} z_{\text {laser }}}{I_{\mathrm{RAMAN}} C_{s}},
$$

where $I_{\text {SERS }}$ is the SERS intensity of the particular peak of NBT while $I_{\text {RAMAN }}$ represents the unenhanced Raman intensity measured in liquid form. $N_{\text {SERS }}$ is the number of NBT molecules on the substrate contributing to the SERS signal and $N_{\text {BULK }}$ is the number of molecules contributing to the unenhanced Raman signal [32]. By incorporating the number of molecules in $\mu \mathrm{m}^{3}\left(N v_{\mathrm{BULK}}\right)$, average height of focused laser beam in $\mu \mathrm{m}\left(z_{\text {laser }}\right)$, and SERS surface concentration, number of molecules per $\mu \mathrm{m}^{2}\left(C_{s}\right)$ EF can be rewritten as the second part of (1). Details of the EF calculation and results are given in Supplementary Materials available online at https://doi.org/10.1155/2017/9182025.

\section{Results and Discussion}

3.1. Performance of AuNP@Al Foil Substrate Tested with Detection of NBT and CV as Compared with AuNPs@Au Film and AuNPs@Glass Substrates. From the data obtained from Raman spectra of the NBT (Figure 1(a)) limit of detection and enhancement factor were determined. We calculated the LOD as a concentration of analyte at three standard deviations of the blank from the plot of blank adjusted signal versus logarithm of analyte concentration.

NBT and CV ware dissolved in acetonitrile and sequentially diluted before drop-casting solutions in a broad concentration range from $0.010 \mathrm{mM}$ to $0.30 \mathrm{nM}$ pM or $\sim 33000$ times. The results were analyzed by plotting the intensity 


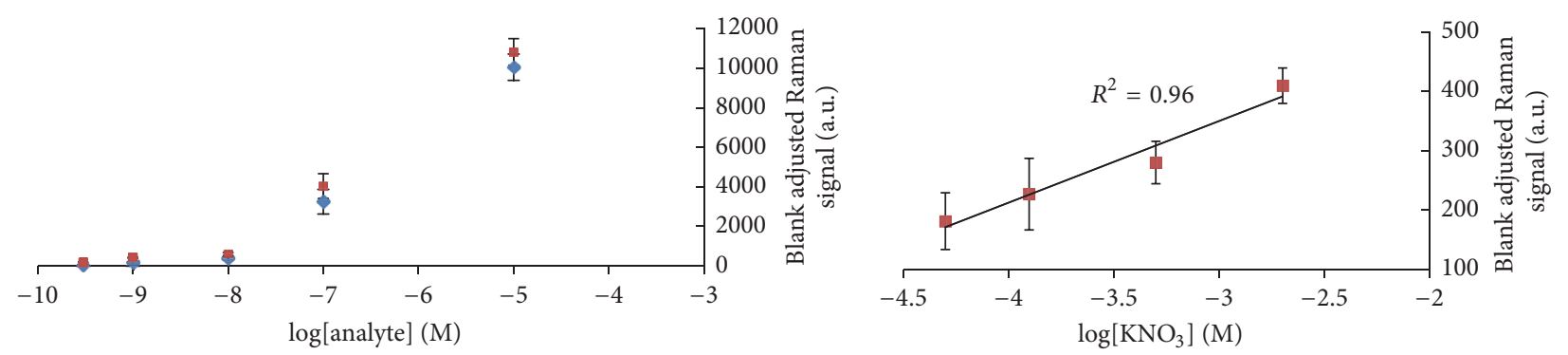

$\rightarrow \mathrm{CV}$

- NBT

(a)

(b)

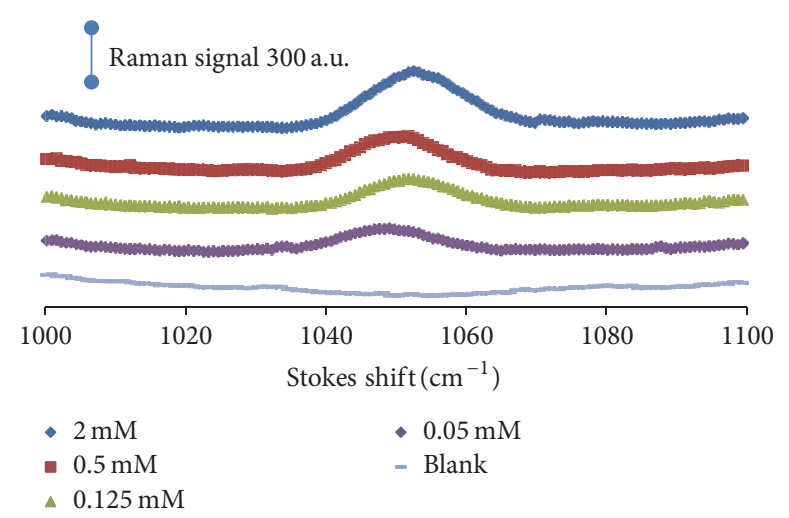

(c)

Figure 2: Plots of blank adjusted Raman signal versus concentrations of analyzed chemicals for SERS assay on unmodified Al foil: (a) for CV and NBT; (b) for $\mathrm{KNO}_{3}$. (c) SERS spectra (vertically adjusted to prevent overlap) for $\mathrm{KNO}_{3}$ at 4 detectable concentrations and blank, taken with $785 \mathrm{~nm}$ laser excitation.

of characteristic stretch vibration $\left(\mathrm{NO}_{2}\right)$ near $1336 \mathrm{~cm}^{-1}$ as a function of the logarithm of total NBT concentration, generating the response curves as one in Figure 2, where signal increased with increase in concentration within broad dynamic range of 8 orders of magnitude.

We got blank adjusted SERS signal of similar magnitude for NBT and CV with $60 \mathrm{~nm}$ AuNPs as shown in Figures 1(b) and 2 and Table 1. Blank adjusted SERS signals for all chemicals are calculated by subtraction of average SERS Raman signal of the blank from average SERS signal of each standard.

As shown in Figure 2(a), both CV and NBT demonstrate a trend of switching to increasing logarithmic sensitivity (slope) in the range about $30-300 \mathrm{nM}$. However, even at lower concentration and lower slope, the linear logarithmic response spans at least two or three orders of magnitude in concentration. The plots of normalized blank adjusted Raman signal of the characteristic peak versus log of analyte concentration are used for calculation of LODs. Those plots are shown for CV and NBT in Figures 1(b) and 1(d) and plot for $\mathrm{KNO}_{3}$ is shown in Figures 2(a) and 2(b). Raman spectra (solid and SERS) in broader range are given for analyzed chemicals in Supplementary Materials. Blank adjusted SERS signals are calculated for all analytes by subtraction of average blank (e.g., solvent: DI water or acetonitrile) signal from average signal of each analyte standard, while both are prepared on the same day and measured with the same parameters.

The maximum analytical enhancement factors (AEF) for the CV and NBT samples at $0.3 \mathrm{nM}$ concentration (above LOD for both chemicals detected on $\mathrm{Al}$ foil) were calculated by (1) above and reported in Table 1 . This table also summarizes LOD and the concentration range used for LOD calculation as well as major parameters of substrate preparation such as AuNP size and number of centrifugation cycles.

The maximum analytical SERS enhancement factors (AEF) above the limit of detection are $6 \times 10^{6}$ for $\mathrm{CV}(0.3 \mathrm{nM})$ with $633 \mathrm{~nm}$ excitation and $1.2 \times 10^{7}$ for NBT $(0.3 \mathrm{nM})$ with $785 \mathrm{~nm}$ excitations. Those magnitudes of EF are on a high end of SERS analytical EFs range and they establish AuNPs@Al foil as potentially strong SERS substrate with unique cost to SERS efficiency ratio.

3.2. Optimization of the Substrate Preparation: Impact of the Centrifugation/Resuspension Cycles. We optimized SERS detection of those chemicals by variation, AuNP size, number of centrifugation cycles, and concentration of AuNP in the applied (drop-casted) solution. The number of centrifugation cycles makes the strongest impact on SERS detection efficiency. For example, as shown in Figure 1 for NBT, three centrifugations versus one centrifugation produce SERS with 
TABLE 1: Raman signals, LODs, and AEFs calculated from detection of CV and detection of NBT on $60 \mathrm{~nm}$ AuNPs set on various substrates (Al foil, Au film, and glass).

\begin{tabular}{|c|c|c|c|c|c|c|c|}
\hline Analyte/substrate & $R^{2}$ & $\begin{array}{c}\text { Slope } \\
\text { signal versus } \\
\log C\end{array}$ & LOD, nM & $\begin{array}{c}C \text { for LOD } \\
\text { (linear range) }\end{array}$ & $\begin{array}{l}\text { Average } \\
\text { Raman } \\
\text { intensity }\end{array}$ & $\begin{array}{c}\text { Standard } \\
\text { error of } \\
\text { Raman signal }\end{array}$ & $\begin{array}{c}\text { AEF for } \\
C=0.3 \mathrm{nM}\end{array}$ \\
\hline \multirow{4}{*}{$\mathrm{CV} / \mathrm{Au}$ film } & \multirow{4}{*}{0,91} & \multirow{4}{*}{166} & \multirow{4}{*}{0.35} & $10 \mathrm{nM}$ & 373 & 87 & \multirow{4}{*}{$3.7 \times 10^{6}$} \\
\hline & & & & $1 \mathrm{nM}$ & 164 & 12 & \\
\hline & & & & $0.3 \mathrm{nM}$ & 131 & 3 & \\
\hline & & & & Blank & 90 & 5 & \\
\hline \multirow{4}{*}{$\mathrm{CV} / \mathrm{Al}$ foil } & \multirow{4}{*}{0,992} & \multirow{4}{*}{2421} & \multirow{4}{*}{0.19} & $10 \mathrm{nM}$ & 494 & 71 & \multirow{4}{*}{$5.5 \times 10^{6}$} \\
\hline & & & & $1 \mathrm{nM}$ & 288 & 21 & \\
\hline & & & & $0.3 \mathrm{nM}$ & 162 & 12 & \\
\hline & & & & Blank & 101 & 4 & \\
\hline \multirow{5}{*}{$\mathrm{CV} /$ glass } & \multirow{5}{*}{0,841} & \multirow{5}{*}{668} & \multirow{5}{*}{1.96} & $100 \mathrm{nM}$ & 1451 & 160 & \multirow{5}{*}{$2.1 \times 10^{6}$} \\
\hline & & & & $10 \mathrm{nM}$ & 164 & 14 & \\
\hline & & & & $1 \mathrm{nM}$ & 115 & 10 & \\
\hline & & & & $0.3 \mathrm{nM}^{*}$ & 116 & 9 & \\
\hline & & & & Blank & 93 & 2 & \\
\hline \multirow{5}{*}{$\mathrm{NBT} / \mathrm{Au}$} & \multirow{5}{*}{0,96} & \multirow{5}{*}{3211} & \multirow{5}{*}{7.23} & $10 \mu \mathrm{M}$ & 10127 & 437 & \multirow{5}{*}{$1.2 \times 10^{7}$} \\
\hline & & & & $1 \mu \mathrm{M}$ & 4124 & 214 & \\
\hline & & & & $100 \mathrm{nM}$ & 388 & 3 & \\
\hline & & & & $0.3 \mathrm{nM}^{*}$ & 438 & & \\
\hline & & & & Blank & 110 & 6 & \\
\hline \multirow{4}{*}{ NBT/Al foil } & \multirow{4}{*}{0,989} & \multirow{4}{*}{265} & \multirow{4}{*}{0.12} & $10 \mathrm{nM}$ & 836 & 45 & \multirow{4}{*}{$2.3 \times 10^{7}$} \\
\hline & & & & $1 \mathrm{nM}$ & 662 & 50 & \\
\hline & & & & $0.3 \mathrm{nM}$ & 410 & 16 & \\
\hline & & & & Blank & 233 & & \\
\hline \multirow{4}{*}{ NBT/glass } & \multirow{4}{*}{0.90} & \multirow{4}{*}{259} & \multirow{4}{*}{0.44} & $10 \mathrm{nM}$ & 608 & 21 & \multirow{4}{*}{$1.5 \times 10^{6}$} \\
\hline & & & & $1 \mathrm{nM}$ & 248 & 3 & \\
\hline & & & & $0.3 \mathrm{nM}$ & 237 & 3 & \\
\hline & & & & Blank & 214 & 3 & \\
\hline
\end{tabular}

$0.3 \mathrm{nM}^{*}$ for CV/glass and $0.3 \mathrm{nM}^{*} \mathrm{NBT} / \mathrm{Au}$ are outside of calibration range, but it shows that $1 \mathrm{nM}$ and $0.3 \mathrm{nM} \mathrm{CV}$ concentrations cannot be distinguished on this substrate.

much higher sensitivity and much lower LOD $(0.1 \mathrm{nM}$ versus $\times 30 \mathrm{nM})$. Overall, two or even three centrifugation cycles produce the best SERS response for this hybrid substrate. The possible explanation is that the layer thickness of stabilizing agent decreases after each centrifugation and the "hot spots" between gold nanoparticles become "hotter" as interparticle distances decrease upon the loss of surfactant during centrifugation/resuspension cycles. Another likely contribution to this increase in SERS signal is that upon the loss of surfactant the gold nanoparticle surface, including hot spots, becomes more accessible for the adsorption of analyte molecules. Data obtained by atomic force microscopy (AFM) with relative standard error in surface concentration 4-19\% are illustrated by representative unprocessed AFM maps in Supplementary Materials. Apparently, the most NPs exist as dimers, trimers, and oligomers (nonsingles count $60-90 \%$ of total number of nanoparticles). Therefore, we would expect a significant contribution to the SERS signal from hot spots generated in the junctions between agglomerated AuNPs. However, as we demonstrated in a recently published study of AuNPs agglomeration on SERS signal with combined AFM Raman imaging the average enhancement on AuNPs nanoparticles dimers is probably higher, but still likely to be on the same order of magnitude as average enhancement on the surface of single AuNPs, when both are laid on metallic film [21].

3.3. Comparison of AuNPs@Al Foil with AuNPs@Gold and AuNPs@Glass in Detection of CV and NBT. Apparently gold nanoparticles generate most of the enhancement in the hybrid substrate because "bare" Al foil (without AuNPs) makes maximum AEF 240 (785 nm excitation) for NBT and $\sim 80$ (633 nm excitation) for CV as shown in AEF calculation table in Supplementary Materials. To compare efficiency of $\mathrm{Al}$ foil as a bottom part of the composite substrate we also did detection of CV and NBT with the same $60 \mathrm{~nm}$ AuNPs on commercial evaporated gold film and AuNPs on glass microscope slide wafer.

Table 1 demonstrates SERS data output obtained with $633 \mathrm{~nm}$ laser excitation in this comparative SERS assay of both Raman labels on 3 different substrates, prepared on the same day with 3 cycles of $60 \mathrm{~nm}$ AuNPs centrifugation/resuspension. 


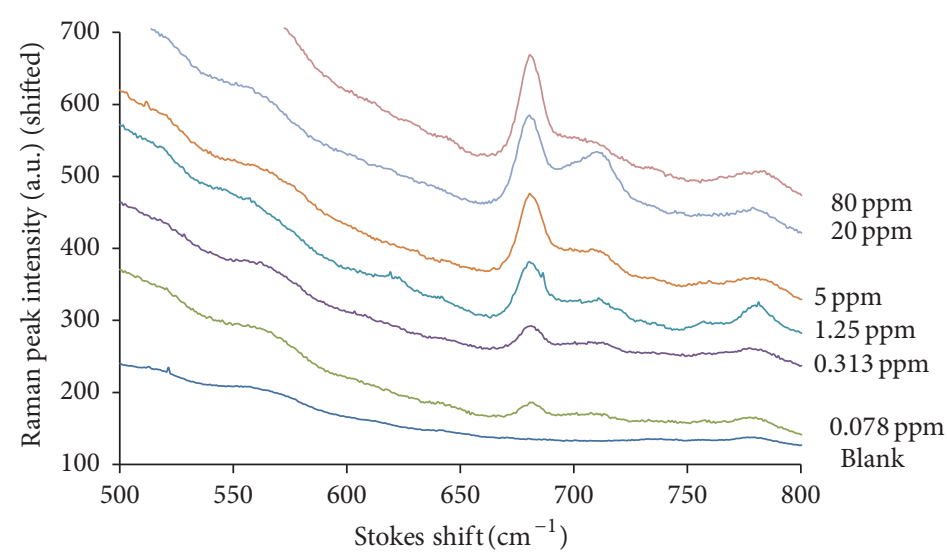

(a)

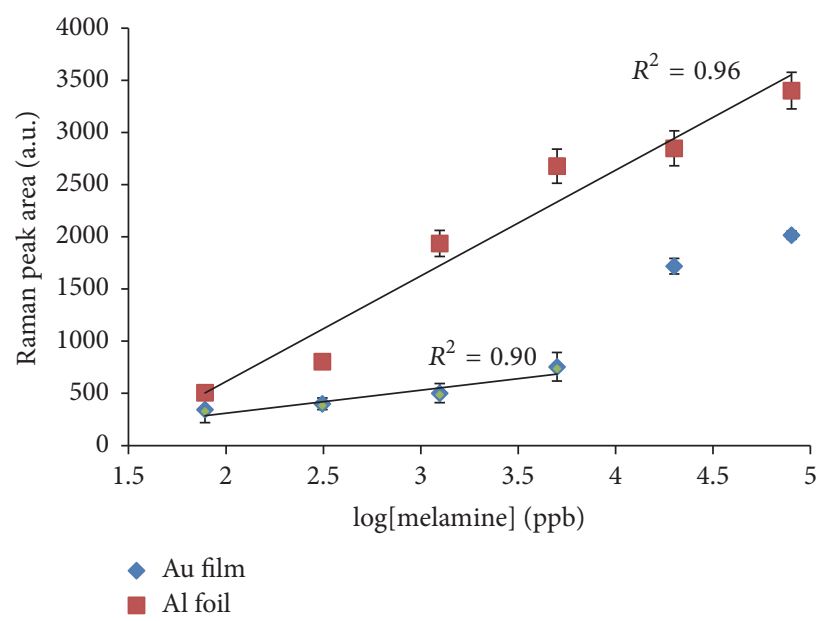

(b)

Figure 3: Detection of melamine. (a) Raman spectra of melamine on $60 \mathrm{~nm}$ AuNPs@Al foil in 0.078 to 80 ppm concentration range, taken with $785 \mathrm{~nm}$ laser excitation. (b) Calibration graph, where $820 \mathrm{~cm}^{-1}$ characteristic vibration peak area is plotted versus $\log C$ in ppb of melamine.

TABLE 2: SERS assay results for KNO3 and paracetamol detection at AuNPs@Al foil substrate.

\begin{tabular}{|c|c|c|c|c|c|c|c|}
\hline Chemicals & Size of AuNPs & Number of CF & $\begin{array}{l}C \text { linear log range } \\
\text { for LOD calc. and } \\
R^{2}\end{array}$ & LOD & $\begin{array}{c}\text { Maximum } \\
\text { AEF }\end{array}$ & $\mathrm{NP} / \mu \mathrm{m}^{2}$ & $\% \mathrm{AG}$ \\
\hline $\mathrm{KNO}_{3}$ & $40 \mathrm{~nm}$ & 2 & $\begin{array}{c}16 \mathrm{mM} \\
0.25 \mathrm{mM} \\
0.961\end{array}$ & $0.7 \mathrm{mM}$ & $2 \times 10^{3}$ & 62 & $91 \%$ \\
\hline
\end{tabular}

$\mathrm{CF}$ is centrifugation/resuspension cycle.

AEF is analytical enhanced factor; \% AG is \% of AuNPs agglomerated (nonsingles).

Table 1 shows that calculated analytical enhancement factors for $0.3 \mathrm{nM} \mathrm{CV}$ and $0.3 \mathrm{nM}$ NBT detected with same $60 \mathrm{~nm}$ AuNPs are substantially higher on Al foil and on gold in comparison with AEF for assays on glass, which is especially the case for detection of NBT, where AEF on gold and $\mathrm{Al}$ foil is multiple of $\times 15$ and $\times 8$, respectively, relative to AEF on glass. The difference between AEF on $\mathrm{Au}$ and $\mathrm{Al}$ is much less significant (within $\times 2$ ). In terms of LOD calculated for CV and NBT alike, Al foil outperformed both gold film and glass as substrates, demonstrating even stronger $\left(R^{2}=0.99\right)$ logarithmic response in the concentration range $0.3-10 \mathrm{nM}$ in comparison to other tested substrates.

3.4. Performance of AuNPs@Al Foil in Detection of Self-Made Explosive Component: $\mathrm{KNO}_{3}$. Table 2 summarizes SERS detection output and AFM characterization results for $\mathrm{KNO}_{3}$ 
and paracetamol. The maximum EF for $\mathrm{KNO}_{3}$ on AuNPs/Al foil is about $2 \times 10^{3}$. This EF is about the same by order of magnitude as $\mathrm{EF}\left(3.6 \times 10^{3}\right)$ reported in the literature for SERS on commercial nanostructured gold Klarite substrate [30].

The obtained LODs and/or EFs on AuNP@Al are at least comparable (or even better) for SERS LODs and/or SERS EFs reported in the literature for those chemicals on other, typically more expensive substrates. For instance, LOD reported for $\mathrm{CV}$ by SERS on $\mathrm{MnO}_{2} / \mathrm{Au}$ hybrid nanowall film was about $10 \mathrm{nM}$ [33]. LOD reported in the assay on concave cubes on Al-6063 alloy (processed for oxide removal) for $\mathrm{CV}$ was about $0.1 \mathrm{nM}[22]$.

\subsection{Detection of Melamine at AuNPs@Al Foil and AuNPs@Au} Film Substrates. We detected melamine in aqueous solution with $60 \mathrm{~nm}$ AuNPs drop-casted on both Al foil and evaporated gold film. The SERS spectra of melamine at various concentrations and calibration plot of the Raman peak area as a function of $\log C$ melamine are shown in Figure 3. The limit of detection of melamine is lower on AuNPs@ Au film (4 ppb) than LOD on AuNPs@Al foil (28 ppb).

However the range of linear response on $\mathrm{Al}$ foil as a substrate is much wider $\left(4^{5}=1280\right)$ than the linear range for the assay on gold film $\left(4^{3}=64\right)$ and this linear responds on $\mathrm{Al}$ foil which has even better linearity with $R^{2}=0.97$ for 6 data points in comparison to the linear response for 4 data points for the detection of melamine on gold film $\left(R^{2}=0.90\right)$.

Therefore, in the assays for melamine Al foil as a part of composite substrate demonstrated its competiveness against more expensive gold film when the same $60 \mathrm{~nm}$ AuNPs are used on both substrates.

Overall the SERS limit of detection on both substrates is by about 1.5-2.5 orders of magnitude lower than the tolerance level recommended for this toxic compound by World Health Organization of $1 \mathrm{ppm}$ [34]. Therefore we demonstrates that SERS on AuNPs@Al foil substrate can confidently detect melamine at concentration well below its legal or health threatening limit.

\section{Conclusion}

In this work, we demonstrated that gold nanoparticles at $\mathrm{Al}$ foil as low-cost hybrid substrate is capable of highly sensitive SERS. Commercial gold nanoparticles on untreated and unmodified $\mathrm{Al}$ foil can be used for subnanomolar detection of Raman active molecules. Various molecules from high end $(\mathrm{CV} ; \mathrm{NBT})$ to low end $\left(\mathrm{KNO}_{3}\right.$; melamine) of SERS enhancement factor range can be detected on AuNPs@Al foil. They are detected on other SERS substrates, including expensive commercial substrates, such as Klarite, with similar magnitude of SERS enhancement and LOD. However, this substrate has advantages over most SERS substrates in simplicity of preparation with no need for sophisticated equipment, low-cost (1-3 cents worth of commercial AuNPs per $1 \mathrm{~mm}^{2}$ of the substrate area), and high availability of the substrate bulk in the form of Al foil. Further work will aim at application of this hybrid low-cost substrate in biosensing.

\section{Conflicts of Interest}

The authors declare that they have no conflicts of interest.

\section{Acknowledgments}

The funding for the research is provided by the Corporate Research Fund of Nazarbayev University.

\section{References}

[1] M. Fleischmann, P. J. Hendra, and A. J. McQuillan, "Raman spectra of pyridine adsorbed at a silver electrode," Chemical Physics Letters, vol. 26, no. 2, pp. 163-166, 1974.

[2] L. Rodriguez-Lorenzo, L. Fabris, and R. A. Alvarez-Puebla, "Multiplex optical sensing with surface-enhanced Raman scattering: a critical review," Analytica Chimica Acta, vol. 745, pp. $10-23,2012$.

[3] T. F. Chen, S. H. Lu, A. J. Wang, D. Zheng, Z. L. Wu, and Y. S. Wang, "Detection of explosives by surface enhanced Raman scattering using substrate with a monolayer of ordered $\mathrm{Au}$ nanoparticles," Applied Surface Science, vol. 317, pp. 940-945, 2014.

[4] D. A. Stuart, K. B. Biggs, and R. P. Van Duyne, "Surfaceenhanced Raman spectroscopy of half-mustard agent," Analyst, vol. 131, no. 4, pp. 568-572, 2006.

[5] W. E. Doering, M. E. Piotti, M. J. Natan, and R. G. Freeman, "SERS as a foundation for nanoscale, optically detected biological labels," Advanced Materials, vol. 19, no. 20, pp. 3100-3108, 2007.

[6] S. Shanmukh, L. Jones, J. Driskell, Y. Zhao, R. Dluhy, and R. A. Tripp, "Rapid and sensitive detection of respiratory virus molecular signatures using a silver nanorod array SERS substrate," Nano Letters, vol. 6, no. 11, pp. 2630-2636, 2006.

[7] K. Kneipp, Y. Wang, H. Kneipp et al., "Single molecule detection using surface-enhanced Raman scattering (SERS)," Physical Review Letters, vol. 78, no. 9, pp. 1667-1670, 1997.

[8] L. Li, T. Hutter, U. Steiner, and S. Mahajan, "Single molecule SERS and detection of biomolecules with a single gold nanoparticle on a mirror junction," Analyst, vol. 138, no. 16, pp. 45744578, 2013.

[9] X. Xia, C. Xie, S. Cai, Z. Yang, and X. Yang, "Corrosion characteristics of copper microparticles and copper nanoparticles in distilled water," Corrosion Science, vol. 48, no. 12, pp. 3924-3932, 2006

[10] B. Sharma, R. R. Frontiera, A.-I. Henry, E. Ringe, and R. P. Van Duyne, "SERS: materials, applications, and the future," Materials Today, vol. 15, no. 1-2, pp. 16-25, 2012.

[11] R. Bukasov, T. A. Ali, P. Nordlander, and J. S. ShumakerParry, "Probing the plasmonic near-field of gold nanocrescent antennas," ACS Nano, vol. 4, no. 11, pp. 6639-6650, 2010.

[12] M. D. Malinsky, K. L. Kelly, G. C. Schatz, and R. P. Van Duyne, "Chain length dependence and sensing capabilities of the localized surface plasmon resonance of silver nanoparticles chemically modified with alkanethiol self-assembled monolayers," Journal of the American Chemical Society, vol. 123, no. 7, pp. 1471-1482, 2001.

[13] G. H. Chan, J. Zhao, G. C. Schatz, and R. P. V. Duyne, "Localized surface plasmon resonance spectroscopy of triangular aluminum nanoparticles," Journal of Physical Chemistry C, vol. 112 , no. 36, pp. 13958-13963, 2008. 
[14] C. Langhammer, M. Schwind, B. Kasemo, and I. Zorić, "Localized surface plasmon resonances in aluminum nanodisks," Nano Letters, vol. 8, no. 5, pp. 1461-1471, 2008.

[15] K. Ray, M. H. Chowdhury, and J. R. Lakowicz, "Aluminum nanostructured films as substrates for enhanced fluorescence in the ultraviolet-blue spectral region," Analytical Chemistry, vol. 79, no. 17, pp. 6480-6487, 2007.

[16] T. Dörfer, M. Schmitt, and J. Popp, "Deep-UV surface-enhanced Raman scattering," Journal of Raman Spectroscopy, vol. 38, no. 11, pp. 1379-1382, 2007.

[17] S. K. Jha, Z. Ahmed, M. Agio, Y. Ekinci, and J. F. Löffler, “DeepUV surface-enhanced resonance Raman scattering of adenine on aluminum nanoparticle arrays," Journal of the American Chemical Society, vol. 134, no. 4, pp. 1966-1969, 2012.

[18] D. O. Sigle, E. Perkins, J. J. Baumberg, and S. Mahajan, "Reproducible deep-UV SERRS on aluminum nanovoids," Journal of Physical Chemistry Letters, vol. 4, no. 9, pp. 1449-1452, 2013.

[19] P. R. West and et al., "Searching for better plasmonic materials," Laser \& Photonics Reviews, vol. 4, no. 6, pp. 795-808, 2010.

[20] K. B. Mogensen, M. Gühlke, J. Kneipp et al., "Surface-enhanced Raman scattering on aluminum using near infrared and visible excitation," Chemical Communications, vol. 50, no. 28, pp. 37443746, 2014.

[21] S. Sergiienko, K. Moor, K. Gudun, Z. Yelemessova, and R. Bukasov, "Nanoparticle-nanoparticle vs. nanoparticle-substrate hot spot contributions to the SERS signal: studying Raman labelled monomers, dimers and trimers," Phys. Chem. Chem. Phys., vol. 19, no. 6, pp. 4478-4487, 2017.

[22] M. M. Martinez-Garcia, P. E. Cardoso-Avila, and J. L. PichardoMolina, "Concave gold nanocubes on Al-6063 alloy as a simple and efficient SERS substrate," Colloids and Surfaces A: Physicochemical and Engineering Aspects, vol. 493, pp. 66-73, 2016.

[23] W. W. Yu and I. M. White, "A simple filter-based approach to surface enhanced Raman spectroscopy for trace chemical detection," Analyst, vol. 137, no. 5, pp. 1168-1173, 2012.

[24] F. H. Scholes, T. J. Davis, K. C. Vernon, D. Lau, S. A. Furman, and A. M. Glenn, "A hybrid substrate for surface-enhanced Raman scattering spectroscopy: coupling metal nanoparticles to strong localised fields on a micro-structured surface," Journal of Raman Spectroscopy, vol. 43, no. 2, pp. 196-201, 2012.

[25] M.-L. Cheng, B.-C. Tsai, and J. Yang, "Silver nanoparticletreated filter paper as a highly sensitive surface-enhanced Raman scattering (SERS) substrate for detection of tyrosine in aqueous solution," Analytica Chimica Acta, vol. 708, no. 1-2, pp. 89-96, 2011.

[26] S. Mabbott, A. Eckmann, C. Casiraghi, and R. Goodacre, “2p or not 2p: tuppence-based SERS for the detection of illicit materials.", The Analyst, vol. 138, no. 1, pp. 118-122, 2013.

[27] I. Šafařík and M. Šafaříková, "Detection of low concentrations of malachite green and crystal violet in water," Water Research, vol. 36, no. 1, pp. 196-200, 2002.

[28] G. Wang, R. J. Lipert, M. Jain et al., "Detection of the potential pancreatic cancer marker MUC4 in serum using surfaceenhanced Raman scattering," Analytical Chemistry, vol. 83, no. 7, pp. 2554-2561, 2011.

[29] B. J. Yakes, R. J. Lipert, J. P. Bannantine, and M. D. Porter, "Detection of Mycobacterium avium subsp. paratuberculosis by a sonicate immunoassay based on surface-enhanced Raman scattering," Clinical and Vaccine Immunology, vol. 15, no. 2, pp. 227-234, 2008.
[30] S. Botti, L. Cantarini, S. Almaviva, A. Puiu, and A. Rufoloni, "Assessment of SERS activity and enhancement factors for highly sensitive gold coated substrates probed with explosive molecules," Chemical Physics Letters, vol. 592, pp. 277-281, 2014.

[31] T. Branigan, Chinese Figures Show Fivefold Rise in Babies Sick from Contaminated Milk, The Guardian, 2008.

[32] W. B. Cai, B. Ren, X. Q. Li et al., "Investigation of surfaceenhanced Raman scattering from platinum electrodes using a confocal Raman microscope: dependence of surface roughening pretreatment," Surface Science, vol. 406, no. 1-3, pp. 9-22, 1998.

[33] M. Zhou, X. Liu, B. Yu et al., " $\mathrm{MnO}_{2} / \mathrm{Au}$ hybrid nanowall film for high-performance surface-enhanced Raman scattering substrate," Applied Surface Science, vol. 333, pp. 78-85, 2015.

[34] T. Lang, S. Pang, and L. He, "Integration of colorimetric and SERS detection for rapid screening and validation of melamine in milk," Analytical Methods, vol. 7, no. 15, pp. 6426-6431, 2015. 

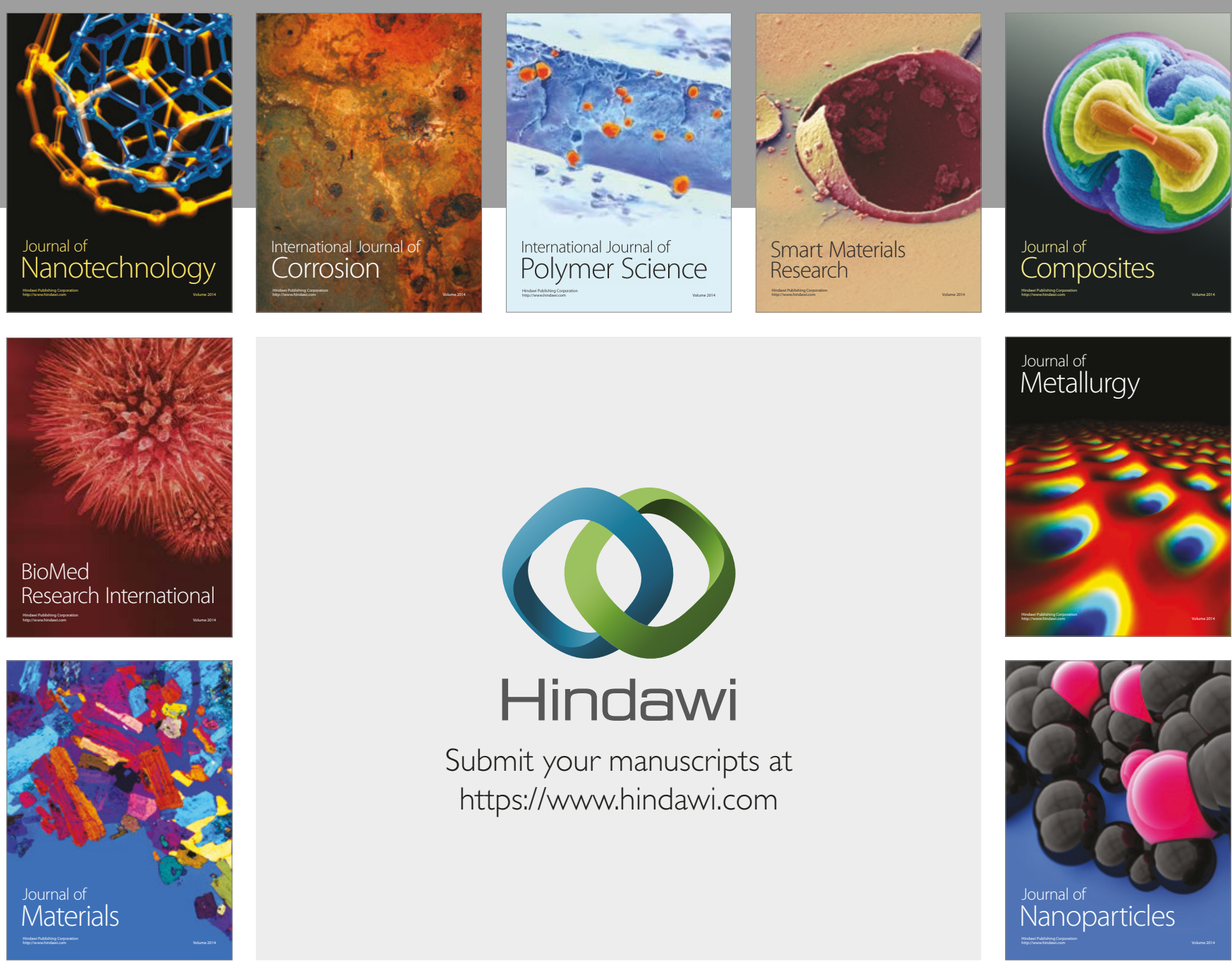

\section{Hindawi}

Submit your manuscripts at

https://www.hindawi.com
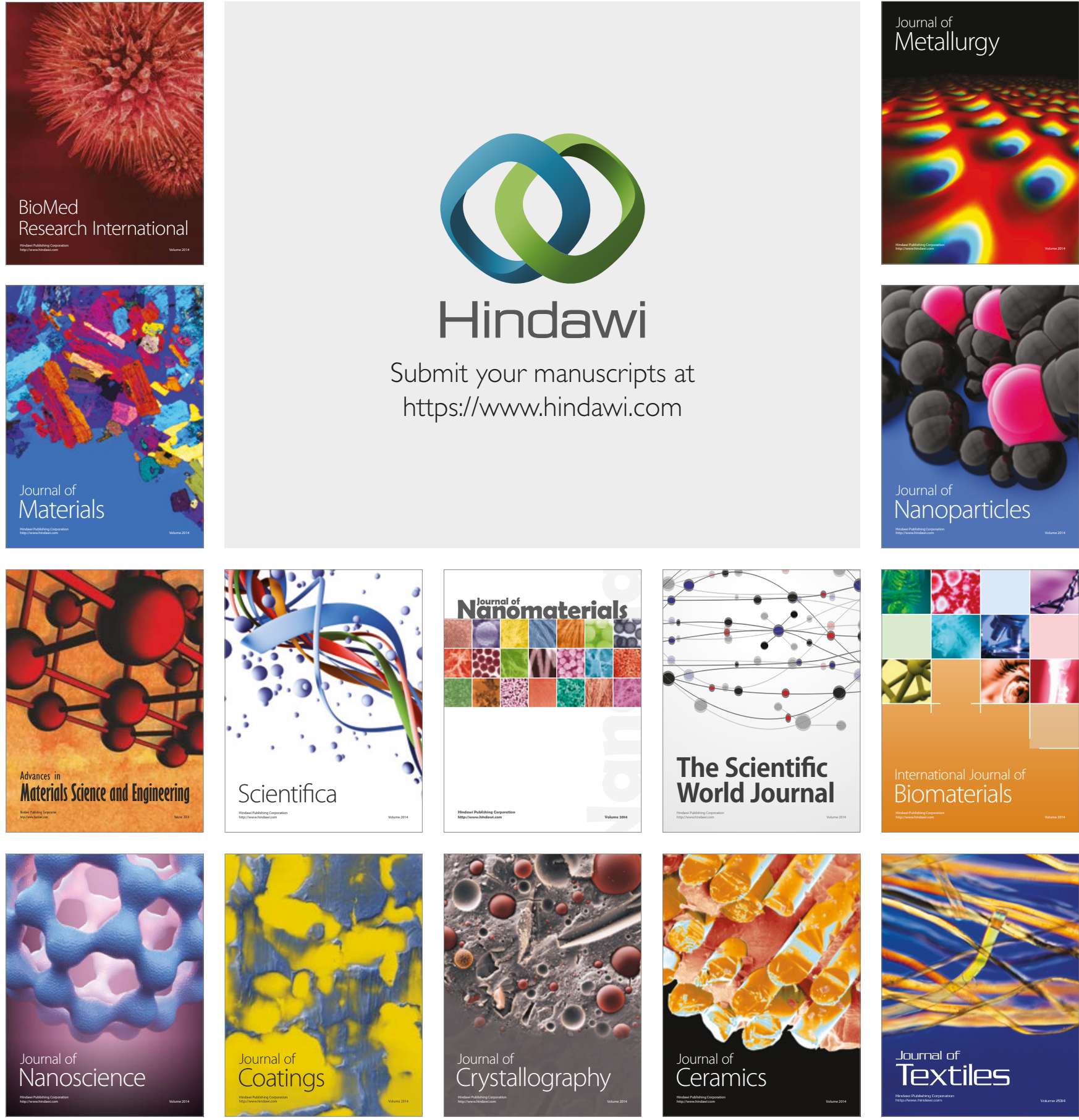

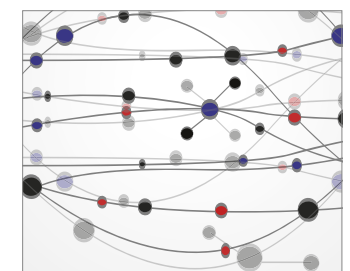

The Scientific World Journal
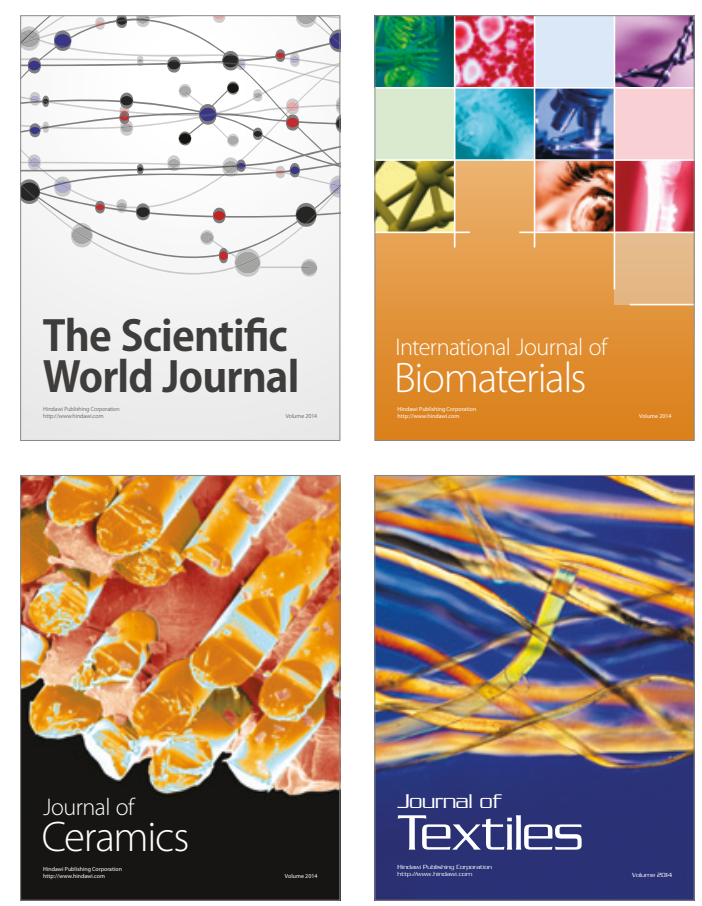